ARTICLE

https://doi.org/10.1038/s41467-021-27480-5

\title{
OPEN
}

\section{Piezoelectricity in hafnia}

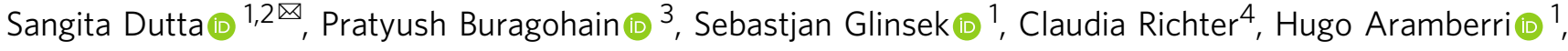

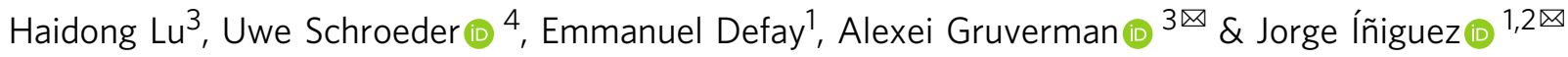

Because of its compatibility with semiconductor-based technologies, hafnia $\left(\mathrm{HfO}_{2}\right)$ is today's most promising ferroelectric material for applications in electronics. Yet, knowledge on the ferroic and electromechanical response properties of this all-important compound is still lacking. Interestingly, $\mathrm{HfO}_{2}$ has recently been predicted to display a negative longitudinal piezoelectric effect, which sets it apart from classic ferroelectrics (e.g., perovskite oxides like $\mathrm{PbTiO}_{3}$ ) and is reminiscent of the behavior of some organic compounds. The present work corroborates this behavior, by first-principles calculations and an experimental investigation of $\mathrm{HfO}_{2}$ thin films using piezoresponse force microscopy. Further, the simulations show how the chemical coordination of the active oxygen atoms is responsible for the negative longitudinal piezoelectric effect. Building on these insights, it is predicted that, by controlling the environment of such active oxygens (e.g., by means of an epitaxial strain), it is possible to change the sign of the piezoelectric response of the material.

\footnotetext{
${ }^{1}$ Materials Research and Technology Department, Luxembourg Institute of Science and Technology, 5 avenue des Hauts-Fourneaux, L-4362 Esch/ Alzette, Luxembourg. ${ }^{2}$ Department of Physics and Materials Science, University of Luxembourg, 41 Rue du Brill, Belvaux L-4422, Luxembourg. ${ }^{3}$ Department of Physics and Astronomy, University of Nebraska-Lincoln, Lincoln, NE 68588-0299, USA. ${ }^{4}$ NaMLab gGmbH, Noethnitzer Strasse 64 a, 01187

Dresden, Germany.凶email: sangita.dutta@list.lu; agruverman2@unl.edu; jorge.iniguez@list.lu
} 
afnia $\left(\mathrm{HfO}_{2}\right)$ is a well-known material in the electronics industry, since its introduction in 2007 by Intel as a convenient gate dielectric for field-effect transistors $(\text { FETs })^{1}$. The announcement of ferroelectricity in this compound a few years later ${ }^{2,3}$ caused great excitement, as it opened the door to the development of (inexpensive, easy to process) electronic devices that could benefit from a switchable polarization, e.g., memories based on ferroelectric FETs. Ever since, a lot of efforts have focused on understanding and controlling ferroelectricity in $\mathrm{HfO}_{2}$, taking advantage of the unique possibilities it may offer (e.g., ferroelectric negative-capacitance effects ${ }^{4}$ ) and advancing towards commercial devices. By now, ferroelectric hafnia has gathered the interest of engineers, materials scientists, and physicists alike, being one of today's best studied and most promising materials.

As compared to traditional soft-mode ferroelectrics (e.g., perovskite oxides like $\mathrm{PbTiO}_{3}$ or $\mathrm{BaTiO}_{3}{ }^{5}$ ), hafnia displays many peculiar features that we are only starting to understand. For example, theoretical work suggests that hafnia's ferroelectricity may not be proper in character ${ }^{6-8}$, yet it is switchable, which sets this compound apart from all ferroelectrics used so far in applications. Further, the nature of its (anti)polar instabilities is such that very narrow domains, and very narrow domain walls, occur naturally in it ${ }^{9}, 10$; in effect, this makes $\mathrm{HfO}_{2}$ a quasi-2D ferroelectric, and may explain the resilience of its polar phase in nanometric samples. In fact, unlike traditional materials, $\mathrm{HfO}_{2}$ seems to improve its ferroelectric properties as the samples decrease in size; moreover, the first reports of ferroelectricity in thick films or bulk samples are very recent ${ }^{11,12}$. In sum, from both applied and fundamental perspectives, ferroelectric hafnia is revealing itself as a very interesting compound.

Comparatively, the electromechanical response properties of ferroelectric $\mathrm{HfO}_{2}$ have received little attention so far, although we believe this situation will quickly change. Indeed, the processing advantages that hafnia offers (as compared to perovskite oxides) make it a viable candidate for applications as a piezoelectric (e.g., in piezotronics, radio-frequency filters) where it might potentially compete with wurtzite compounds (e.g., AlN, $\mathrm{ZnO}$ ).

At a more fundamental level, it has been recently predicted from first principles ${ }^{13,14}$ that the usual ferroelectric phase of $\mathrm{HfO}_{2}$ (orthorhombic with space group $P c a 2_{1}$ ) presents a negative longitudinal piezoresponse, i.e., that compressing the material along the polarization direction will result in an enhancement of its polar distortion. If confirmed, this property would widen even more the gap between $\mathrm{HfO}_{2}$ and the ferroelectric perovskite oxides, all of which behave in exactly the opposite way ${ }^{15}$. Intriguingly, though, existing experimental measurements of hafnia's piezoresponse $^{11}$ suggest a perovskite-like behavior (i.e., a positive longitudinal effect), and thus contradict the first-principles predictions. Further, we still lack a satisfying physical picture explaining the atomistic origin of the predicted negative piezoresponse, a simple understanding that would allow us to propose ways to control and optimize the effect. Hence, in our opinion, the piezoelectric response of hafnia is an open problem.

Here we present a first-principles and experimental investigation of the piezoelectric properties of $\mathrm{HfO}_{2}$. First, we confirm the negative longitudinal effect, from both theory and experiment, notwithstanding the fact that the experimental result is sample dependent. Second, based on our first-principles simulations, we provide a simple and plausible explanation of the atomistic mechanisms controlling the effect. Further, based on this understanding, we predict that the ferroelectric phase of hafnia can be modified (by epitaxial strain) to either enhance or reduce the negative longitudinal piezoresponse, or even change its sign. We conclude with a brief discussion of the implications of our results, and an outlook of the challenges and opportunities ahead.

\section{Results and discussion}

In the following we present our simulation and experimental results. In most occasions we discuss in parallel our findings for $\mathrm{HfO}_{2}$ and the corresponding results for a representative ferroelectric perovskite, which allows us to better highlight the specificity of hafnia as compared with classic materials. For the perovskite, we consider $\mathrm{PbTiO}_{3}$ at the theoretical level and $\mathrm{PbZr}_{1-x} \mathrm{Ti}_{x} \mathrm{O}_{3}(\mathrm{PZT}$, with $x=0.6)$ at the experimental level. In our experimental presentation, we also show results for polyvinylidene fluoride (PVDF), a compound with a wellcharacterized negative longitudinal piezoresponse ${ }^{16,17}$.

Basic first-principles predictions. We start our first-principles investigation by relaxing the most usual ferroelectric polymorph of $\mathrm{HfO}_{2}$, with $\mathrm{Pca}_{1}$ space group. The obtained solution (Supplementary Table 1) agrees well with previous results in the literature ${ }^{18,19}$. The ferroelectric polarization characterizing the $P c a 2_{1}$ phase can be appreciated by comparing the cubic paraelectric structure of Fig. 1a with the polar state in Fig. 1b: it is caused by the downward shift of the $\mathrm{O}_{\mathrm{I}}$ anion sublattice, which results in a positive polarization along the third Cartesian direction ( $c$ axis in the figure). Note that all Hf cations in the

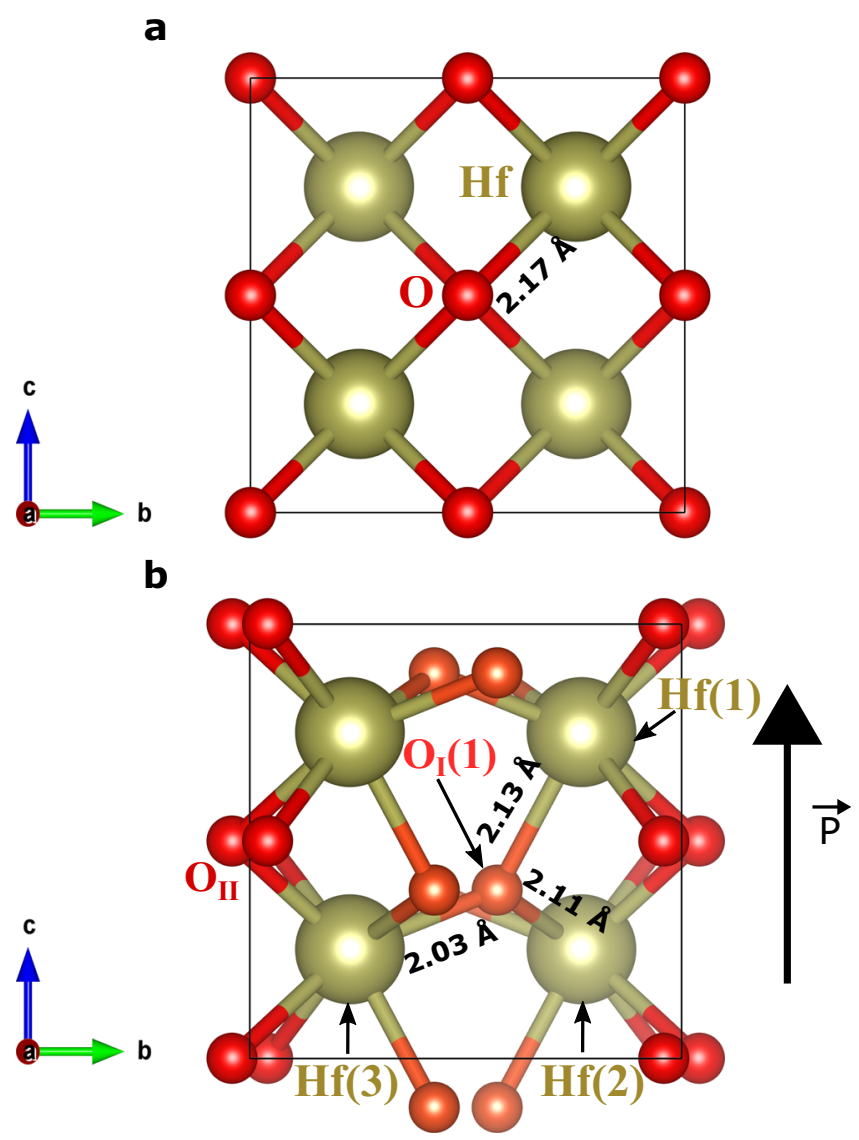

Fig. 1 Key structural features of $\mathbf{H f O}_{\mathbf{2}}$. Structure of the cubic paraelectric (a) and orthorhombic ferroelectric (b) polymorphs of $\mathrm{HfO}_{2}$. In the cubic $\mathrm{Fm} \overline{3} \mathrm{~m}$ phase, all $\mathrm{Hf}$ and $\mathrm{O}$ atoms are equivalent by symmetry. In the ferroelectric $P c a 2_{1}$ structure we have two symmetry-inequivalent sets of oxygen atoms -labeled $\mathrm{O}_{1}$ (shown in orange) and $\mathrm{O}_{11}$ (red), respectively-, while all $\mathrm{Hf}$ atoms are equivalent. In panel $\mathbf{b}$, the black arrow indicates the positive spontaneous polarization of the structure shown, which is essentially related to the vertical downward shift of the $\mathrm{O}_{1}$ atoms from their high-symmetry position in the cubic phase. In the ferroelectric state, the $\mathrm{O}_{1}$ atoms have three nearest-neighboring $\mathrm{Hf}$ cations; the bonding distances are explicitly indicated for the $\mathrm{O}_{1}(1)$ atom, which is a representative case. 


\begin{tabular}{|lcccc|}
\multicolumn{5}{|c|}{ Table 1 Calculated piezoelectric tensors for $\mathbf{H f O}_{\mathbf{2}}$. } \\
& Index & $\overline{\mathbf{e}}$ & $\mathbf{e}$ & $\boldsymbol{d}$ \\
\hline VASP & 31 & -0.37 & -1.31 & -1.71 \\
& 32 & -0.34 & -1.33 & -1.77 \\
& 33 & 0.62 & -1.44 & -2.51 \\
& 15 & -0.28 & -0.20 & -2.03 \\
& 24 & -0.20 & 0.64 & 6.74 \\
ABINIT & 31 & -0.39 & -1.53 & -2.71 \\
& 32 & -0.36 & -1.40 & -1.66 \\
& 33 & 0.65 & -1.34 & -1.64 \\
& 15 & -0.29 & -0.23 & -2.70 \\
& 24 & -0.22 & 0.69 & 6.60 \\
\hline
\end{tabular}

We show the total (e) and frozen-ion (e) direct piezoelectric tensor (in $\mathrm{C}^{-2}$ ), as well as the total converse piezoelectric tensor $\mathbf{d}$ (in $\mathrm{pm} \mathrm{V}^{-1}$ ). Indices given in Voigt notation.

polar phase are equivalent by symmetry and occupy a Wyckoff position that we label 'Hf. In contrast, the polar state features two symmetry-inequivalent oxygen sublattices, which we label ' $\mathrm{O}_{\mathrm{I}}$ ' and ' $\mathrm{O}_{\mathrm{II}}$ ', respectively. Also, in Fig. $1 \mathrm{~b}$ and the following we use a number in parenthesis (e.g., ' $\mathrm{Hf}(2)$ ' or ' $\mathrm{O}_{\mathrm{I}}(1)$ ') to label individual atoms belonging to a particular sublattice.

To fix ideas, noting that the sign of the piezoelectric coefficients depends on the sign of the spontaneous polarization ${ }^{20}$, in the following we always work with the ferroelectric state with positive polarization as shown in Fig. 1b. We calculate this polarization to be $P_{3}=54.75 \mu \mathrm{C} \mathrm{cm}{ }^{-2}$, in agreement with previous literature ${ }^{18,19}$.

We now consider the piezoelectric tensor ${ }^{21}$

$$
e_{\alpha j}=\frac{\partial P_{\alpha}}{\partial \eta_{j}}
$$

where $P_{\alpha}$ is the polarization component along Cartesian direction $\alpha$ and $\eta_{j}$ is a symmetric strain labeled using Voigt notation ${ }^{21}$. For analysis purposes, it is convenient to decompose the piezoelectric response into a frozen-ion contribution $\left(\bar{e}_{\alpha j}\right)$ and a latticemediated part (defined as the difference $e_{\alpha j}-\bar{e}_{\alpha j}$ ). $\bar{e}_{\alpha j}$ is obtained by freezing the atoms in their unperturbed equilibrium positions, so they cannot respond to the applied strain; it thus captures a purely electronic effect. Finally, from knowledge of the $e_{\alpha j}$ and the elastic constants $C_{j k}$, one can obtain

$$
d_{\alpha j}=\frac{\partial P_{\alpha}}{\partial \sigma_{j}}=\left(C^{-1}\right)_{j k} e_{\alpha k}=S_{j k} e_{\alpha k}
$$

where $\sigma_{j}$ is the $j$-th component of an applied external stress (in Voigt notation), $\mathbf{S}=\mathbf{C}^{-1}$ is the elastic compliance, and we assume summation over repeated indices. Note that this piezoresponse $\mathbf{d}$ tensor is of interest, as it is the one most easily accessible in experiment and the one usually exploited in applications. Computing the piezoelectric tensors is straightforward using density functional perturbation theory (DFPT) ${ }^{22}$.

Table 1 shows the results we obtain for $\mathbf{e}, \overline{\mathbf{e}}$, and $\mathbf{d}$ using two different-but essentially equivalent, both accurate-implementations of density functional theory (DFT). (We attribute the existing numerical differences mainly to the use of different pseudopotentials; see the Experimental Section.) We confirm a negative value of the $e_{33}$ coefficient, indicating that a positive strain (stretching) of the unit cell along the polar direction will yield a reduction of the polarization. (Recall that we work with an unperturbed state with $P_{3}>0$ and $P_{1}=P_{2}=0$.) We further verify this result by performing a finite-difference calculation of the change in $P_{3}$ upon application of a small strain $\eta_{3}$. Our results are also in agreement with the DFT predictions previously published $^{14}$.
Table 2 Same as Table 1, but for the ferroelectric phase of $\mathrm{PbTiO}_{3}$.

\begin{tabular}{lrll} 
Index & $\overline{\mathbf{e}}$ & $\mathbf{e}$ & $\boldsymbol{d}$ \\
\hline 31 & 0.23 & 1.62 & -39 \\
33 & -0.30 & 4.95 & 208 \\
15 & 0.03 & 4.58 & 78 \\
\hline
\end{tabular}

It is interesting to note that the lattice-mediated response is always larger than the frozen-ion response. In particular, Table 1 clearly shows that the lattice response is responsible for the negative value of $e_{33}$.

Supplementary Table 2 shows the results obtained for the elastic and compliance tensors, which allow us to compute the d tensor in Table 1 . While the relationship between $\mathbf{e}$ and $\mathbf{d}$ is not trivial in materials with a relatively low symmetry (as is the case of ferroelectric $\mathrm{HfO}_{2}$ ), we do obtain a negative $d_{33}=-2.51 \mathrm{pm}$ $\mathrm{V}^{-1}$. Hence, we predict that, upon application of a compressive stress $\sigma_{3}<0, P_{3}$ will increase.

Table 2 shows results for the piezoelectric tensors of the ferroelectric phase of $\mathrm{PbTiO}_{3}$ (tetragonal, with space group $\mathrm{P} 4 \mathrm{~mm}$ ). We find that the $e_{\alpha j}$ coefficients are generally larger for the perovskite than for $\mathrm{HfO}_{2}$ (by a factor of up to 4, if we focus on their absolute values). Interestingly, the difference becomes much greater for the $d_{\alpha j}$ coefficients, as $\mathrm{PbTiO}_{3}$ presents values between 1 and 2 orders of magnitude larger than those of hafnia: for example, we get a $d_{33}$ of $208 \mathrm{pm} \mathrm{V}^{-1}$ for $\mathrm{PbTiO}_{3}$ and $-2.51 \mathrm{pm} \mathrm{V}-1$ for $\mathrm{HfO}_{2}$. The obtained giant $d_{33}$ response of lead titanate agrees with previous experimental ${ }^{23}$ and theoretical ${ }^{24}$ reports. Interestingly, our calculations indicate that the main reason behind this result is the softness of the compound along the polarization direction: we get $C_{33}=51.8$ $\mathrm{GPa}$ and $S_{33}=48.72 \mathrm{TPa}^{-1}$ for $\mathrm{PbTiO}_{3}$ (the full tensors are given in Supplementary Table 3 ), which contrast with the much stiffer case of $\mathrm{HfO}_{2}\left(S_{33}=2.97 \mathrm{TPa}^{-1}\right.$, see Supplementary Table 2).

When considering the experimental manifestation of the negative $d_{33}$ predicted for hafnia, one has to take into account an important feature of most samples: they are polycrystalline. Hence, typically, the measured piezoelectric response will not correspond to a unique well-defined orientation, but to an effective average. If we assume a sample composed of randomly oriented grains, and poled so that all grains display a polarization with a positive $P_{3}$ component, we can estimate an effective $d_{33 \text {,eff }}$ as $^{25,26}$

$$
\begin{aligned}
d_{33, \mathrm{eff}} & =\left\langle\cos \theta\left[\left(d_{15}+d_{31}\right)\left(\sin ^{2} \theta \sin ^{2} \varphi\right)+\left(d_{24}+d_{32}\right)\left(\sin ^{2} \theta \cos ^{2} \varphi\right)+d_{33} \cos ^{2} \theta\right]\right\rangle \\
& =\left(d_{15}+d_{31}\right)\left\langle\cos \theta \sin ^{2} \theta\right\rangle\left\langle\sin ^{2} \varphi\right\rangle+\left(d_{24}+d_{32}\right)\left\langle\cos \theta \sin ^{2} \theta\right\rangle\left\langle\cos ^{2} \varphi\right\rangle+d_{33}\left\langle\cos ^{3} \theta\right\rangle \\
& =\frac{1}{3 \pi}\left(d_{15}+d_{31}+d_{24}+d_{32}\right)+\frac{4}{3 \pi} d_{33},
\end{aligned}
$$

where $\langle\ldots\rangle$ indicates an average over the Euler angles $\varphi$ and $\theta$, which span all possible orientations with $P_{3}>0$ (i.e., $0<\theta<\pi / 2$ and $0<\varphi<2 \pi)$. By calculating this average we obtain $d_{33 \text {,eff }}=$ $-0.94 \mathrm{pm} \mathrm{V} \mathrm{V}^{-1}$, suggesting that even in polycrystalline $\mathrm{HfO}_{2}$ samples we expect to measure a negative longitudinal piezoresponse. Hence, to summarize: according to our DFT calculations, the $d_{33}$ response of $\mathrm{HfO}_{2}$ is predicted to be somewhere between $-2.5 \mathrm{pm} \mathrm{V}^{-1}$ (single-crystal limit) and $-0.9 \mathrm{pm} \mathrm{V}^{-1}$ (untextured fully poled polycrystalline limit).

Experimental confirmation. To determine experimentally the sign of the effective piezoelectric coefficient $d_{33 \text {,eff }}$ in hafnia, we carry out comparative dynamic piezoelectric measurements by means of piezoresponse force microscopy (PFM), using materials with known piezoelectric coefficients as a reference. In PFM, 
a

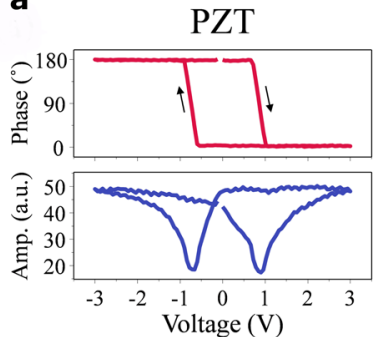

b

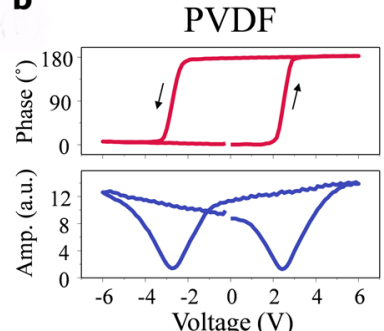

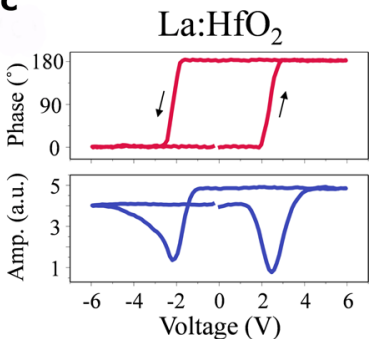

Fig. 2 Dynamic measurements of piezoelectricity using piezoresponse force microscopy (PFM). a-c PFM phase (top panel) and amplitude (bottom panel) loops measured in the $\mathrm{IrO}_{2} / \mathrm{PZT} / \mathrm{Pt}$ capacitor (a), PVDF film (b), and Pt/Ti/TiN/La:HfO $/$ TiN capacitor (c). The loops were obtained in the bias-off mode to minimize the electrostatic contribution to the PFM signal.

application of an alternating (ac) electric field to the sample via a conductive tip results in an oscillation with the frequency of the applied field, due to the converse piezoelectric effect ${ }^{27,28}$. The amplitude and phase of the oscillation provide information about

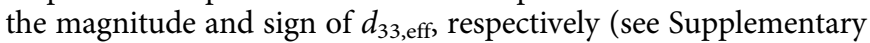
Note 1 and Supplementary Figs. 1-3).

In a material with a positive longitudinal piezoresponse, such as $\mathrm{PZT}^{29}$, the sample oscillation will be in phase with the driving electric field when the polarization is oriented downward, while it will be in anti-phase when the polarization is oriented upward. In addition, in the switching spectroscopy mode of PFM, a pulsed low-frequency triangular waveform is superimposed on the ac waveform to generate a piezoelectric strain hysteresis loop related to the local polarization reversal ${ }^{30}$. Thus, the sense of rotation of the PFM phase loops in Fig. 2 is directly related to the sign of the

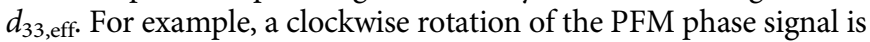
indicative of a positive $d_{33 \text {,eff }}$ coefficient, as is illustrated by the PFM phase hysteresis loop measured in the $\mathrm{IrO}_{2} / \mathrm{PZT} / \mathrm{Pt}$ capacitor in Fig. 2a. In this case, the phase signal is in phase (anti-phase) with the ac modulation field at the far positive (negative) dc bias, which generates the downward (upward) orientation of the polarization. In contrast, in a material with a negative $d_{33 \text {,eff, such }}$ as PVDF 17,31 , the phase signal is in phase (anti-phase) with the ac modulation field at the far negative (positive) dc bias corresponding to the upward (downward) orientation of the polarization, resulting in anti-clockwise rotation in the PFM phase loop (Fig. 2b). (See the Experimental Section for details on our PZT and PVDF samples). Under the same conditions, as shown in Fig. 2c, the PFM phase loop measured in TiN/La: $\mathrm{HfO}_{2} / \mathrm{TiN}$ capacitors (with $20 \mathrm{~nm}$-thick $\mathrm{La}: \mathrm{HfO}_{2}$ ) exhibits an anti-clockwise rotation similar to that of PVDF films and opposite to that of PZT capacitors. (See the Experimental Section for details on our $\mathrm{HfO}_{2}$ samples; note that similar results were obtained for $10 \mathrm{~nm}$-thick samples.) Clearly, this behavior is indicative of the negative sign of $d_{33 \text {,eff }}$ in the $\mathrm{La}: \mathrm{HfO}_{2}$ film. We estimate $d_{33 \text {,eff }}$ to be between -2 $\mathrm{pm} \mathrm{V}^{-1}$ and $-5 \mathrm{pm} \mathrm{V}^{-1}$ (Supplementary Fig. 2), in excellent agreement with our theoretical result between $-0.9 \mathrm{pm} \mathrm{V}^{-1}$ (in the polycrystalline disordered limit) and $-2.5 \mathrm{pm} \mathrm{V}^{-1}$ (in the single-crystal limit).

It is important to note here that evidence for a positive longitudinal piezoresponse $d_{33 \text {,eff }}$ in hafnia has been reported in the experimental literature, for example, for ultrathin $(10 \mathrm{~nm}) \mathrm{Si}$ doped $\mathrm{HfO}_{2}$ films ${ }^{32}$, for thicker $(70 \mathrm{~nm}) \mathrm{Y}$-doped films ${ }^{33}$, or for La-doped films with thicknesses up to $1 \mu \mathrm{m}^{11}$. Indeed, in the course of this work, we found ourselves that the application of the same experimental protocol to other $\mathrm{HfO}_{2}$ films (thicker, grown by different means) yields a positive longitudinal effect. Hence, one may wonder what is especial about the $\mathrm{La}: \mathrm{HfO}_{2}$ films for which we get a negative effect. May it be extrinsic?

Interestingly, the $\mathrm{La}: \mathrm{HfO}_{2}$ films studied here were characterized in ref. ${ }^{34}$ using grazing-incidence X-ray diffraction. In that work, it was determined that these $\mathrm{La}: \mathrm{HfO}_{2}$ films present a strong outof-plane texture, leading to relatively high remnant polarization compared to other dopants. This stronger texture may cause local strains in the films, and potentially act as an extrinsic factor affecting the local electromechanical properties. However, since all the domains measured in our study showed a negative response, we tend to believe that the effect observed in this work is intrinsic.

As far as we can tell, there is only one other publication suggesting a similar negative longitudinal piezoresponse. In 2019 Chouprik et al. ${ }^{35}$ reported 'anomalous' switching in $10 \mathrm{~nm}$-thick films of $\mathrm{Hf}_{0.5} \mathrm{Zr}_{0.5} \mathrm{O}_{2}$, which in PFM appeared as a polarization reversal against the applied electric field, and could thus be interpreted as a negative piezoresponse. This behavior was observed in about $20 \%$ of the domains of the pristine films.

Hence, there is experimental evidence that the piezoelectric properties of $\mathrm{HfO}_{2}$ are sample and sample-history dependent. This suggests that a careful and systematic characterization will be needed to determine the factors (intrinsic or extrinsic) controlling the piezoresponse, including its sign.

Origin of the negative piezoresponse. Our DFPT calculations allow us to track down the computed negative value of $e_{33}$. As presented in Supplementary Note 2, within perturbation theory 22 we write the piezoelectric tensor as

$$
e_{\alpha j}=\bar{e}_{\alpha j}+\Omega_{0}^{-1} Z_{m \alpha}\left(\Phi^{-1}\right)_{m n} \Lambda_{n j},
$$

where the second term on the right-hand side of this equation shows that the lattice-mediated part of e depends on the unit-cell volume of the unperturbed system $\left(\Omega_{0}\right)$, the Born effective charge tensor $(\mathbf{Z}$, which quantifies the polarization change caused by atomic displacements), the force-constant matrix ( $\Phi$, i.e., the second derivatives of the energy with respect to atomic displacements) and the force-response internal strain tensor $(\boldsymbol{\Lambda}$, which quantifies the atomic forces that appear when a strain is applied). Here, $m$ is a combined index that runs over all atoms in the unit cell and the three spatial directions.

By inspecting the calculated tensors for $\mathrm{HfO}_{2}$, and by comparing with those obtained for $\mathrm{PbTiO}_{3}$, we can identify the atomistic underpinnings of the sign of $e_{33}$.

First off, let us note that there is nothing peculiar concerning the force-constant matrices $\boldsymbol{\Phi}$ : for both materials, these matrices reflect the fact that the ferroelectric phase is a stable equilibrium state. Hence, they are positively defined tensors without any feature that is relevant to the present discussion.

As for the Born effective charges $\mathbf{Z}$ (Supplementary Table 4), they have the expected signs and are relatively large in magnitude: we get values over +5 for $\mathrm{Hf}$ and below -2.5 for $\mathrm{O}$, exceeding the nominal respective charges of +4 and -2 . This feature reflects a mixed ionic-covalent character of the chemical bonds in the material, and is typical of other ferroelectrics like, e.g., $\mathrm{PbTiO}_{3}$ 
Table $3 \Lambda$ tensors for the symmetry-inequivalent atoms of the ferroelectric phase of $\mathrm{HfO}_{2}$ (in $\mathrm{eV} \AA^{-1}$ ).

$\begin{aligned} \mathrm{Hf} & {\left[\begin{array}{cccccc}-4.02 & 0.00 & -2.29 & 8.39 & 0.83 & 0.79 \\ -1.04 & 11.52 & -1.40 & 4.50 & 3.58 & 5.87 \\ -1.87 & 1.45 & -\mathbf{4 . 0 7} & -2.83 & -2.61 & 3.42\end{array}\right] } \\ \mathrm{O}_{\mathrm{I}} & {\left[\begin{array}{ccccccc}2.06 & -0.63 & 1.09 & -5.87 & -3.29 & 0.91 \\ -1.97 & -1.75 & -1.43 & -2.77 & -4.60 & -2.34 \\ -1.61 & 0.42 & \mathbf{3 . 2 2} & 1.36 & 0.29 & -4.53\end{array}\right] } \\ \mathrm{O}_{\mathrm{II}} & {\left[\begin{array}{ccccccc}-5.87 & 1.10 & -1.07 & 4.67 & 2.46 & 0.06 \\ 0.79 & 7.87 & 0.54 & -1.71 & 4.10 & 0.52 \\ 3.46 & 1.86 & \mathbf{0 . 8 5} & 1.27 & -1.36 & 5.44\end{array}\right] }\end{aligned}$

The 3 rows correspond, respectively, to the 3 spatial directions; the 6 columns correspond respectively, to the 6 strain indices in Voigt notation. Marked in bold are the entries controlling the longitudinal piezoresponse, as discussed in the text.

itself (see the Born charges we obtain for $\mathrm{PbTiO}_{3}$ in Supplementary Table 5). In addition, because of the relatively low site symmetries in the ferroelectric phase of $\mathrm{HfO}_{2}$, in this compound, the charge tensors present small non-zero off-diagonal components. While this feature does set $\mathrm{HfO}_{2}$ apart from $\mathrm{PbTiO}_{3}$, we checked it has no influence in the sign of $e_{33}$. In conclusion, the $\mathbf{Z}$ tensors do not explain the differentiated behavior of $e_{33}$ in these two compounds.

Hence, we are left with the $\boldsymbol{\Lambda}$ tensors, which are given in Table 3 and Supplementary Table 6 for $\mathrm{HfO}_{2}$ and $\mathrm{PbTiO}_{3}$, respectively. Let us focus on the " 33 " entries for each of the atomspecific tensors, i.e., the numbers quantifying the atomic force along direction 3 (parallel to the polarization) caused by a positive strain $\eta_{3}>0$ (stretching). In the case of $\mathrm{PbTiO}_{3}$, the straininduced forces are positive for the cations $(\mathrm{Pb}$ and $\mathrm{Ti})$ and negative for the two symmetry-inequivalent oxygens in the unit cell. This means that, in response to the vertical stretching of the cell, the cations will tend to move up while the oxygens will tend to move down. Since the unperturbed state has $P_{3}>0$, this movement will clearly yield an increase of the polarization; hence, we have $e_{33}>0$.

The situation is just opposite for $\mathrm{HfO}_{2}$ : in this case, a stretching of the cell $\left(\eta_{3}>0\right)$ causes the Hf cations to move down and the oxygens (particularly those of type I) to move up. Since the starting point has $P_{3}>0$, and since the Born charges in $\mathrm{HfO}_{2}$ have the natural signs for cations and anions, these strain-induced displacements will yield a reduction of the magnitude of the polarization. This is indeed reflected in our computed $e_{33}<0$; we have thus identified the atomistic origin of the effect.

Physical insight. While the above discussion is clear from a numerical point of view, it hardly provides us with a satisfying physical understanding. Can we rationalize the mechanisms controlling the sign of the strain-induced forces and, thus, of $e_{33}$ ?

In perovskite oxides, it is known that the structural instabilities of the parent cubic phase (as, e.g., those leading to ferroelectricity) are largely determined by steric and ion-size aspects, usually discussed in terms of simple descriptors such as the Goldschmidt tolerance factor ${ }^{36}$. Ultimately, these effects are a reflection of the ions' tendency to optimize the chemical bonds in their first (nearest neighbor) coordination shell, as successfully captured by phenomenological theories such as, for example, the bondvalence model $^{37}$.

These bonding considerations readily allow us to understand the piezoelectric response in $\mathrm{PbTiO}_{3}$, a simple model case. In this compound, the cubic paraelectric phase presents $\mathrm{Ti}$ and $\mathrm{Pb}$ cations that are equidistant to 6 and 12 first-neighboring oxygens, respectively. Then, as shown in Fig. 3 (for a state with $P_{3}>0$ ), the ferroelectric distortion results in a tetragonal structure where the cations reduce the number of closest oxygens neighbors. For the sake of simplicity, let us focus on the case of the central Ti cation, which passes from being 6-fold coordinated in the paraelectric phase (panel a) to having only 5 close oxygens in the ferroelectric state (panel b). In fact, among these oxygens, there is one (the apical type I oxygen that lies above Ti) forming the shortest (and strongest) $\mathrm{Ti}-\mathrm{O}$ bond. (We know the details of this bond from previous theoretical works on $\mathrm{PbTiO}_{3}$, which also show that the type II oxygens are mainly bonded to the $\mathrm{Pb}$ cations $\mathrm{s}^{38}$.) Imagine we now stretch the cell along the polarization direction $\left(\eta_{3}>0\right)$, and assume that the atoms will rearrange in order to maintain the preferred length of the strongest bonds. For that to happen, as sketched in Fig. $3 c$, the central $\mathrm{Ti}$ should move up and the mentioned $\mathrm{O}_{\mathrm{I}}$ oxygen that bonds with it should move down, which results in an increased polarization. This expectation is in perfect correspondence with our computed $\Lambda$ tensor (see Supplementary Table 4). A similar argument applies to the displacements of the $\mathrm{Pb}$ and $\mathrm{O}_{\mathrm{II}}$ ions in reaction to $\eta_{3}>0$. Thus, this simple picture explains the positive $e_{33}$ obtained for $\mathrm{PbTiO}_{3}$.

The situation in $\mathrm{HfO}_{2}$ is harder to analyze, for two main reasons. First, the atomic chemical environments are far more complex than in $\mathrm{PbTiO}_{3}$ and identifying dominant bonds is not trivial. (Our attempts at a clear-cut quantification-e.g., by inspecting the magnitude of the interatomic force constantswere not convincing enough.) Second, as shown from first principles ${ }^{6-8}$, the nature of ferroelectricity in $\mathrm{HfO}_{2}$ is far more complicated than in $\mathrm{PbTiO}_{3}$. Nevertheless, an appealing physical picture emerges from our results, as follows.

Let us start by inspecting the distortions connecting the cubic paraelectric phase (a convenient reference for the sake of this argument) and the $P c a 2_{1}$ ferroelectric state of $\mathrm{HfO}_{2}$. In the cubic phase, all $\mathrm{O}$ anions have four nearest-neighboring $\mathrm{Hf}$ cations, and all $\mathrm{Hf}-\mathrm{O}$ bond distances are equal to $2.17 \AA$. In contrast, in the ferroelectric phase we have two sublattices of symmetryequivalent oxygens (labeled $\mathrm{O}_{\mathrm{I}}$ and $\mathrm{O}_{\mathrm{II}}$ ), and all oxygens form relatively short bonds with only three Hf atoms. Let us focus on the $\mathrm{O}_{\mathrm{I}}$ sublattice, responsible for the spontaneous polarization. As shown in Fig. $1 \mathrm{~b}$ for the representative case of the $\mathrm{O}_{\mathrm{I}}(1)$ atom, we have the following shortest bonding distances: $2.13 \AA$ for $\mathrm{Hf}(1)-\mathrm{O}_{\mathrm{I}}(1), 2.11 \AA$ for $\mathrm{Hf}(2)-\mathrm{O}_{\mathrm{I}}(1)$, and $2.03 \AA$ for $\mathrm{Hf}(3)-\mathrm{O}_{\mathrm{I}}(1)$. Further, the computed equilibrium charge density in Fig. $4 \mathrm{a}, \mathrm{b}$ suggests that $\mathrm{O}_{\mathrm{I}}(1)$ forms similarly strong bonds with its three neighboring $\mathrm{Hf}$ cations.

It is thus apparent that the formation of a reduced number of relatively short $\mathrm{Hf}-\mathrm{O}$ bonds is the driving force for the stabilization of the ferroelectric state. However, interestingly, the atomic rearrangements yielding the preferred Hf-O coordination do not necessarily contribute to the spontaneous polarization. For example, as indicated in Fig. 3d, e, to reach the optimal configuration the $\mathrm{O}_{\mathrm{I}}$ anions shift both vertically (parallel to the polar axis) and horizontally (perpendicular to it). The former displacements yield the spontaneous polarization of $\mathrm{HfO}_{2}$, while the latter (which follow an anti-polar pattern of sorts) have no contribution to it. As for the $\mathrm{O}_{\text {II }}$ anions, Fig. 3e shows that they present anti-polar displacements along the horizontal direction, with no contribution to the polarization. These observations suggest that the development of the spontaneous polarization may not be the main driving force for the $P c a 2_{1}$ phase to occur. Indeed, first-principles calculations show that the cubic phase of $\mathrm{HfO}_{2}$ does not present any ferroelectric instability ${ }^{6}$. Additional first-principles studies suggest that the occurrence of the $P c a 2_{1}$ phase of $\mathrm{HfO}_{2}$ depends on the prior condensation of a non-polar mode that constitutes a strong instability of the cubic state (and yields a well-known tetragonal polymorph), and that it is further conditioned by a 


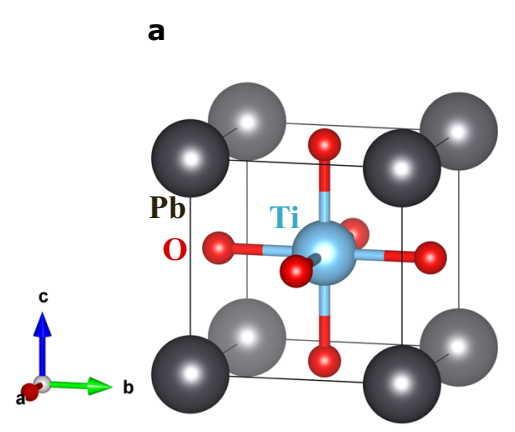

b
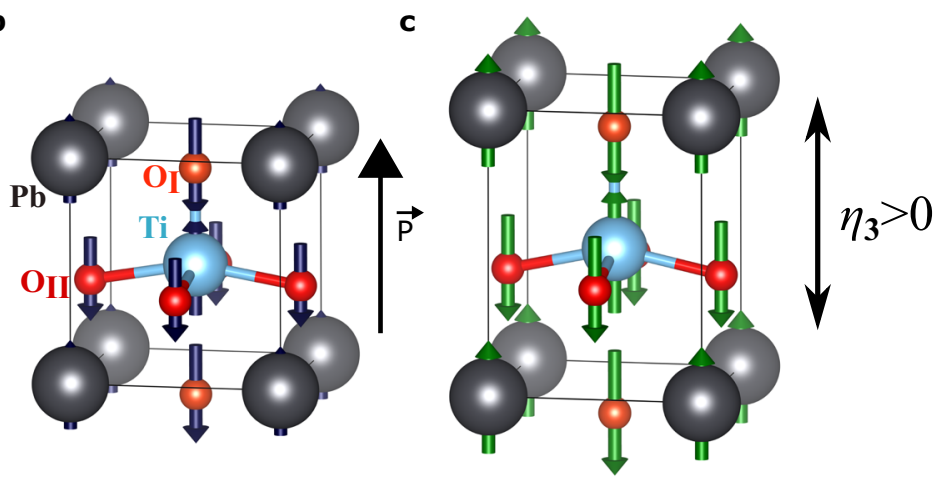

d

e

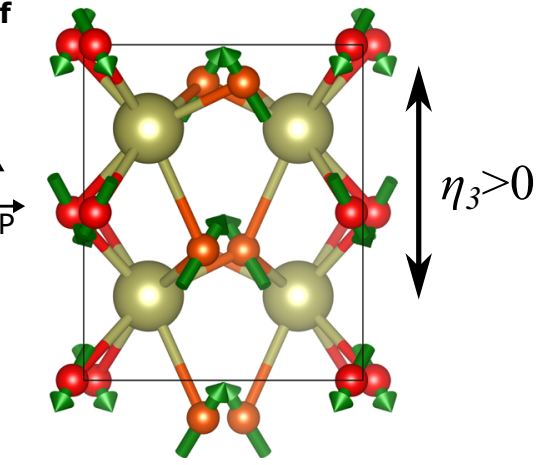

Fig. 3 Comparison between $\mathbf{P b T i O}_{\mathbf{3}}$ and $\mathbf{H f O}_{\mathbf{2}}$. Cubic $\mathrm{Pm} \overline{3} \mathrm{~m}$ paraelectric (a) and tetragonal $\mathrm{P} 4 \mathrm{~mm}$ ferroelectric (b) phases of $\mathrm{PbTiO}$. The tetragonal phase presents two symmetry-inequivalent oxygen anions, colored differently and labeled by $\mathrm{O}_{1}$ and $\mathrm{O}_{\|}$, respectively. In panel $\mathbf{b}$ the arrow on the right marks the spontaneous polarization, which is essentially related to the upward displacement of the $\mathrm{Pb}$ and Ti cations with respect to the oxygen atoms (the arrows on the atoms mark such displacements). Panel $\mathbf{c}$ is a sketch of the tetragonal phase subject to a tensile $\eta_{3}>0$ strain (the strain is exaggerated for clarity); the arrows on the atoms indicate how they react in response to the strain, as computed from first principles. Panels $\mathbf{d}-\mathbf{f}$ are analogous to the previous three panels, but featuring the paraelectric (d) and ferroelectric (e) states of $\mathrm{HfO}_{2}$, and its longitudinal piezoresponse (f).

very strong coupling between the polarization and other supplementary (hard) distortions ${ }^{7,8}$. Hence, $\mathrm{HfO}_{2}$ is qualitatively different from proper ferroelectric perovskites like $\mathrm{PbTiO}_{3}$.

Then, to understand the $e_{33}$ piezoresponse of $\mathrm{HfO}_{2}$, we have to discuss how a strain along the polar axis will affect the position of the $\mathrm{O}_{\mathrm{I}}$ ions responsible for the spontaneous polarization. By inspection of the atomic environment of the $\mathrm{O}_{\mathrm{I}}(1)$ atom (a representative case), we make a critical observation: there is only one $\mathrm{Hf}-\mathrm{O}_{\mathrm{I}}(1)$ bond clearly aligned with the polar axis, namely, the one connecting $\mathrm{O}_{\mathrm{I}}(1)$ with the $\mathrm{Hf}(1)$ cation above it (Fig. 1b); this bond will be strongly affected by a vertical $\eta_{3}$ strain. In contrast, the remaining two $\mathrm{Hf}-\mathrm{O}_{\mathrm{I}}(1)$ bonds, with $\mathrm{Hf}(2)$ and $\mathrm{Hf}(3)$, lie largely in the horizontal $a b$ plane, and will be mildly impacted by a vertical strain. Thus, upon application of a tensile strain $\eta_{3}>0$, we can expect $\mathrm{O}_{\mathrm{I}}(1)$ to move upward in order to preserve the optimum $\mathrm{Hf}(1)-\mathrm{O}_{\mathrm{I}}(1)$ distance. This is exactly the behavior we find from first principles, illustrated in Fig. 3f. Interestingly, and somewhat anecdotally, the upward movement of $\mathrm{O}_{\mathrm{I}}(1)$ (Fig. 3f) goes against the downward shift of this very same ion when the polar phase condenses (Fig. 3e). Consequently, the polarization is reduced in reaction to $\eta_{3}>0$, which yields a negative $e_{33}$.

The difference between $\mathrm{PbTiO}_{3}$ and $\mathrm{HfO}_{2}$ is pictorically illustrated in Fig. 3: in $\mathrm{PbTiO}_{3}$ the ionic reaction to $\eta_{3}>0$ (panel c) adds to the distortion responsible for the spontaneous polarization (panel d), while in $\mathrm{HfO}_{2}$ it goes largely against it (for $\mathrm{O}_{\mathrm{I}}$, the arrows of panel e are reversed in panel f). Ultimately, it is the peculiar chemical environment of the $\mathrm{O}_{\mathrm{I}}$ anions in $\mathrm{HfO}_{2}$ that is responsible for the effect.

While appealing, this picture may seem speculative. Nevertheless, it suggests that, by controlling the chemical environment of the $\mathrm{O}_{\mathrm{I}}$ atoms that dominate the $e_{33}$ response, we may be able to affect the magnitude of the effect in a very definite way. More precisely: by decreasing the $\mathrm{Hf}(1)-\mathrm{O}_{\mathrm{I}}(1)$ distance, we may be able to make this bond stronger and, thus, make $e_{33}$ more negative; conversely, by weakening the $\mathrm{Hf}(1)-\mathrm{O}_{\mathrm{I}}(1)$ link, we should have a response increasingly controlled by the $\mathrm{Hf}(2)-\mathrm{O}_{\mathrm{I}}(1)$ and $\mathrm{Hf}(3)-\mathrm{O}_{\mathrm{I}}(1)$ pairs, which should result in a less negative $e_{33}$. We put this hypothesis to a test in the next section.

Prediction of a tunable piezoresponse. To control the bonds of interest and monitor their effect on $e_{33}$, we simulate the $P c a 2_{1}$ ferroelectric phase of $\mathrm{HfO}_{2}$ subject to an isotropic epitaxial strain in the plane perpendicular to the polarization.

We do this by running structural relaxations where the inplane lattice vectors are constrained to form a $90^{\circ}$ angle and their magnitudes fixed to $a=a_{0}\left(1+\eta_{\mathrm{epi}}\right)$ and $b=b_{0}\left(1+\eta_{\mathrm{epi}}\right)$, where $a_{0}$ and $b_{0}$ are the previously obtained equilibrium lattice constants (Supplementary Table 1) and $\eta_{\text {epi }}$ the applied epitaxial strain. Our calculations suggest that the $P c a 2_{1}$ orthorhombic phase is an equilibrium energy minimum in a wide $\eta_{\mathrm{epi}}$-range, from about $-7 \%$ to about $+4 \%$. (We find that beyond this range the ferroelectric polymorph losses its stability and transforms into other structures that are of no interest here.) Figure 5 shows our results for the $\eta_{\text {epi }}$ dependence of the Hf- $\mathrm{O}_{\mathrm{I}}(1)$ bond lengths, which follow the expected behavior: the $\mathrm{Hf}(1)-\mathrm{O}_{\mathrm{I}}(1)$ link (which largely lies along the vertical direction) gets longer as we compress in-plane $\left(\eta_{\text {epi }}<0\right)$, following the growth of the out-ofplane lattice constant $c$ (see Supplementary Fig. 4). Conversely, the $\mathrm{Hf}(2)-\mathrm{O}_{\mathrm{I}}(1)$ and $\mathrm{Hf}(3)-\mathrm{O}_{\mathrm{I}}(1)$ bonds (which are essentially perpendicular to the vertical polar axis) shrink upon in-plane compression. We have thus achieved the desired control on the atomic environment of the $\mathrm{O}_{\mathrm{I}}$ atoms. (The analogous results for 
a

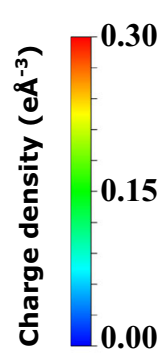

C

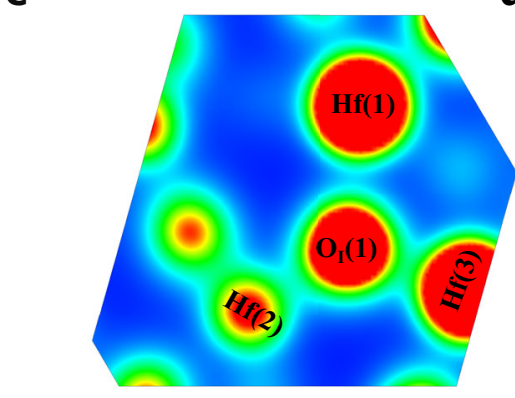

e

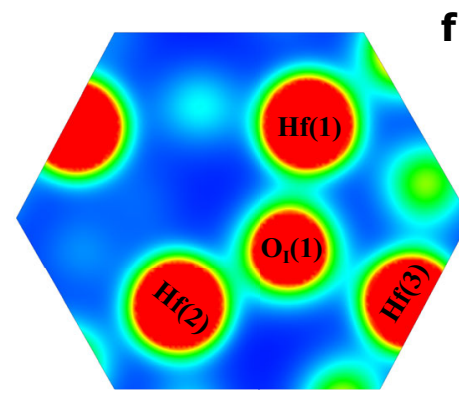

b
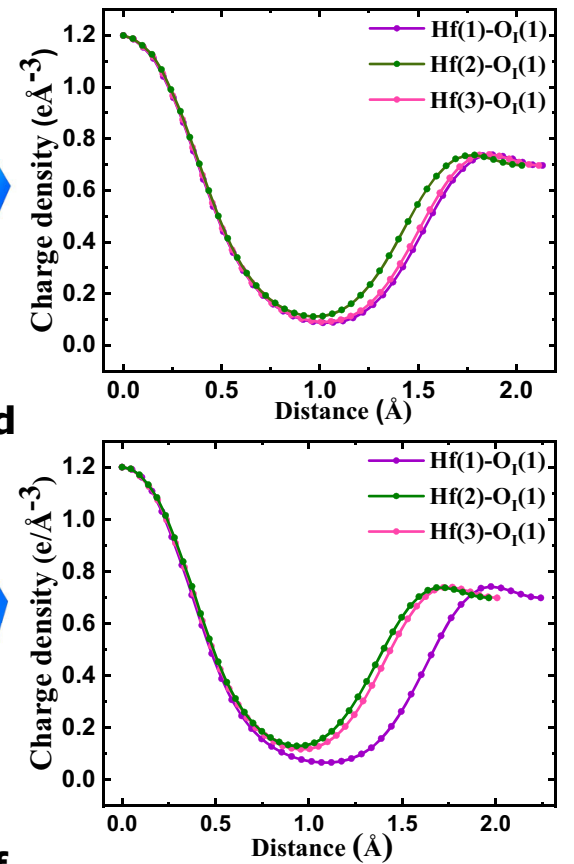

f

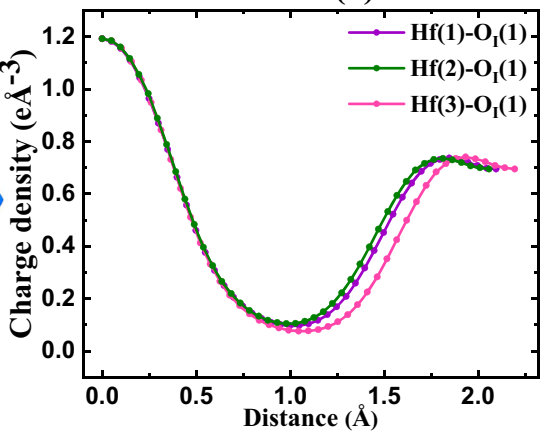

Fig. 4 Visualizing Hf-O bonds. Computed electronic charge density for the unperturbed ferroelectric phase of $\mathrm{HfO}_{2}$ (panels a and $\mathbf{b}$ ) as well as for the structures obtained at $\eta_{\mathrm{epi}}=-7 \%$ ( $\mathbf{c}$ and $\left.\mathbf{d}\right)$ and $\eta_{\mathrm{epi}}=+4 \%$ (e and $\mathbf{f}$ ). Panels $\mathbf{a}, \mathbf{c}$ and $\mathbf{e}$ show a contour plot of the charge density within a plane that approximately contains the $\mathrm{O}_{1}(1)$ atom highlighted in Fig. 1 as well as its three nearest-neighboring $\mathrm{Hf}$ atoms. Panels $\mathbf{b}, \mathbf{d}$ and $\mathbf{f}$ show the charge density along lines connecting the central oxygen with each of its three nearest-neighboring $\mathrm{Hf}$ cations. In panel $\mathbf{c}$, the red globe at the top left of the $\mathrm{Hf}(2)$ atom corresponds to a neighboring oxygen anion that gets close to the shown plane.

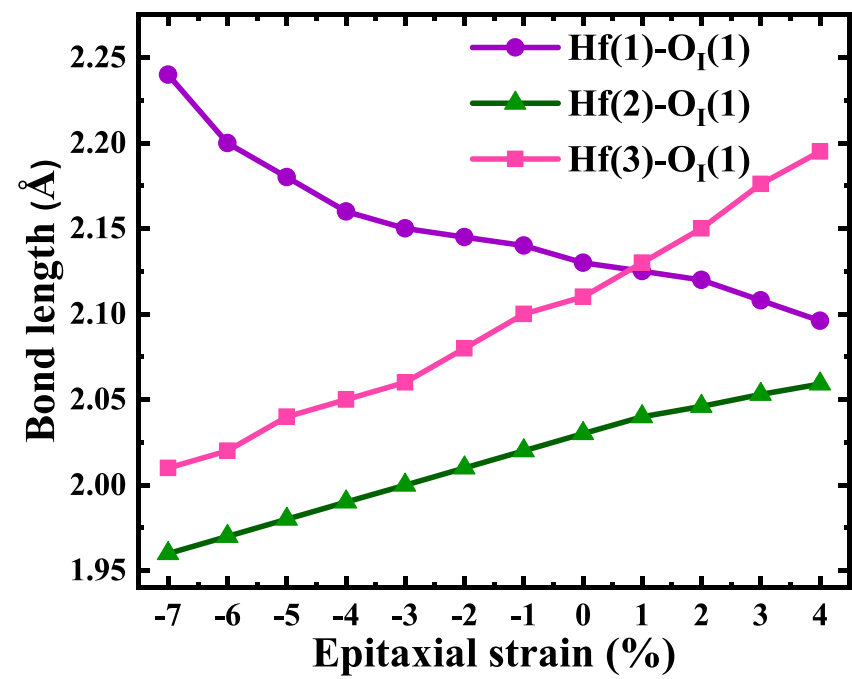

Fig. 5 Controlling bonds with epitaxial strain. Lengths of the $\mathrm{Hf}(1)-\mathrm{O}_{1}(1)$, $\mathrm{Hf}(2)-\mathrm{O}_{1}(1)$ and $\mathrm{Hf}(3)-\mathrm{O}_{1}(1)$ bonds defined in Fig. 1, computed as a function of epitaxial strain. the Hf-O bonds of the $\mathrm{O}_{\text {II }}$ atoms are shown in Supplementary Fig. 5.)

Figure 6 shows the evolution of the $e_{33}$ piezoresponse component, as a function of $\eta_{\text {epi }}$, obtained from DFPT calculations exactly as in the bulk case. We find that the frozen-ion contribution $\bar{e}_{33}$ remains nearly constant (and positive) in the whole range of strains. In contrast, the latticemediated part of the response (red line in the Figure) changes very markedly in a monotonic way. As a result, the total $e_{33}$ changes as well: it reaches its strongest negative response at tensile strains $\left(\eta_{\text {epi }}>0\right)$ and eventually switches to positive values as we compress the material in-plane!

Let us stress that this change of sign in $e_{33}$ occurs even though we have a positive $P_{3}>0$ for all considered $\eta_{\text {epi }}$ values. Indeed, as shown in Supplementary Fig. 6, we find that the polarization grows beyond $70 \mu \mathrm{C} \mathrm{cm}^{-2}$ for epitaxial compression over $-5 \%$, an evolution that is perfectly consistent with that of the structural distortions ( $\mathrm{Hf}-\mathrm{O}_{\mathrm{I}}(1)$ bonds in Fig. 5). Hence, the longitudinal piezoresponse changes sign even though the material remains in the same polar state; as far as we know, such an effect had never been observed (or predicted) before in a ferroelectric. 


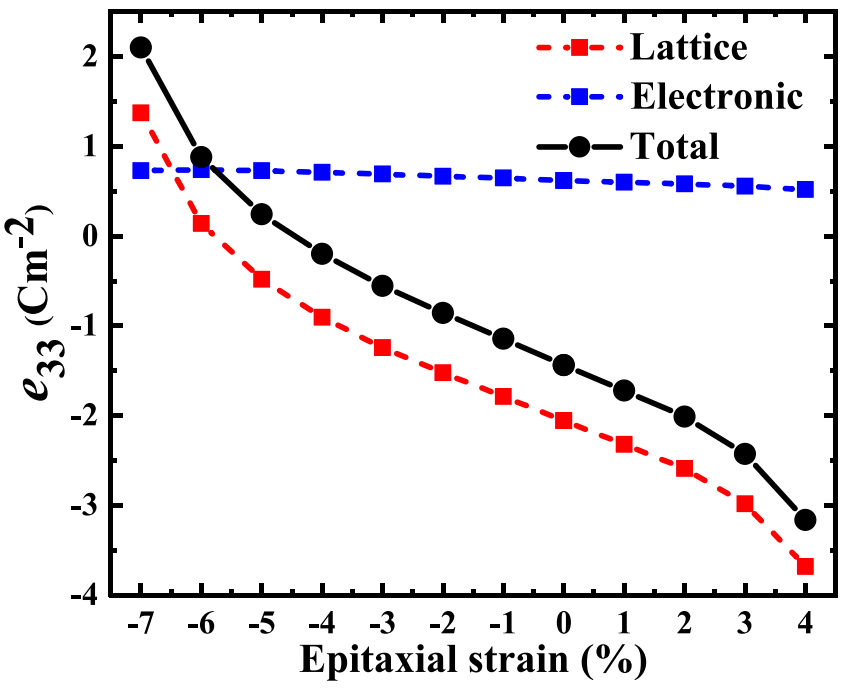

Fig. 6 Computed $e_{33}$ piezoresponse component as a function of epitaxial strain. The total $e_{33}$ (black) is split into frozen-ion (blue) and latticemediated (red) contributions.

We can easily track down the sign change of $e_{33}$ to the key components of the force-response internal-strain tensor, whose evolution with $\eta_{\text {epi }}$ is shown in Fig. 7. At zero strain, we have the situation already discussed above: when we stretch $\left(\eta_{3}>0\right)$ the state with $P_{3}>0$, the system's response involves $\mathrm{O}_{\mathrm{I}}$ anions moving up (the corresponding $\Lambda_{n j}$ component is positive) and all $\mathrm{Hf}$ cations moving down (negative $\Lambda_{n j}$ component), which results in a reduction of the polarization $P_{3}$. Then, as we compress in-plane $\left(\eta_{\text {epi }}<0\right)$, the signs of the $\mathrm{O}_{\mathrm{I}}$ and Hf displacements eventually reverse, and so does the piezoresponse to $\eta_{3}$. Figure 7 also displays the key $\Lambda_{n j}$ component for the $\mathrm{O}_{\mathrm{II}}$ anions; the associated response-relatively small and largely independent of the epitaxial strain-does not play any role in the sign change of $e_{33}$.

Finally, and most importantly, we can check whether our physical picture for the sign of $e_{33}$ is correct. The key results are given in Fig. 4c-f, which shows the computed charge density as obtained for the limit cases with $\eta_{\text {epi }}=-7 \%$ (panels $c$ and d) and $\eta_{\text {epi }}=+4 \%$ (panels e and $\mathrm{f}$ ).

The result in Fig. 4c, d is particularly clear. For strong in-plane compression, the $\mathrm{Hf}(1)-\mathrm{O}_{\mathrm{I}}(1)$ bond is all but broken, as consistent with the long interatomic distance shown in Fig. 5. This suggests that, in this limit, the piezoresponse of the material will be controlled by the $\mathrm{Hf}(2)-\mathrm{O}_{\mathrm{I}}(1)$ and $\mathrm{Hf}(3)-\mathrm{O}_{\mathrm{I}}(1)$ links. Based on this assumption, we expect that a stretching along the vertical direction will result in the $\mathrm{O}_{\mathrm{I}}(1)$ anion moving down and the Hf cations moving up, so that these two bond lengths change as little as possible. This is exactly what we find in our response calculations; and this behavior results in the obtained $e_{33}>0$.

In the other limit $\left(\eta_{\text {epi }}=+4 \%\right.$, Fig. $\left.4 \mathrm{e}, \mathrm{f}\right)$ we find that the $\mathrm{Hf}(1)-\mathrm{O}_{\mathrm{I}}(1)$ and $\mathrm{Hf}(2)-\mathrm{O}_{\mathrm{I}}(1)$ bonds remain strong, while the $\mathrm{Hf}(3)-\mathrm{O}_{\mathrm{I}}(1)$ seems relatively weak. Hence, this case is similar to the bulk-like situation discussed above and corresponding to $\eta_{\text {epi }}=0 \%$. The only difference is that the preponderance of the vertical $\mathrm{Hf}(1)-\mathrm{O}_{\mathrm{I}}(1)$ bond can be expected to grow, which should result in a stronger $e_{33}<0$, as we indeed obtain.

Hence, our epitaxial-strain calculations confirm that the proposed physical understanding of the longitudinal piezoresponse of $\mathrm{HfO}_{2}$ is essentially correct: the peculiar atomic environment of the active oxygen atoms, and the tendency to maintain the optimal length of the dominant $\mathrm{Hf}-\mathrm{O}_{\mathrm{I}}$ bonds, determine the sign of $e_{33}$. Our calculations also show that the value of $e_{33}$ is strongly tunable, and can even change sign,

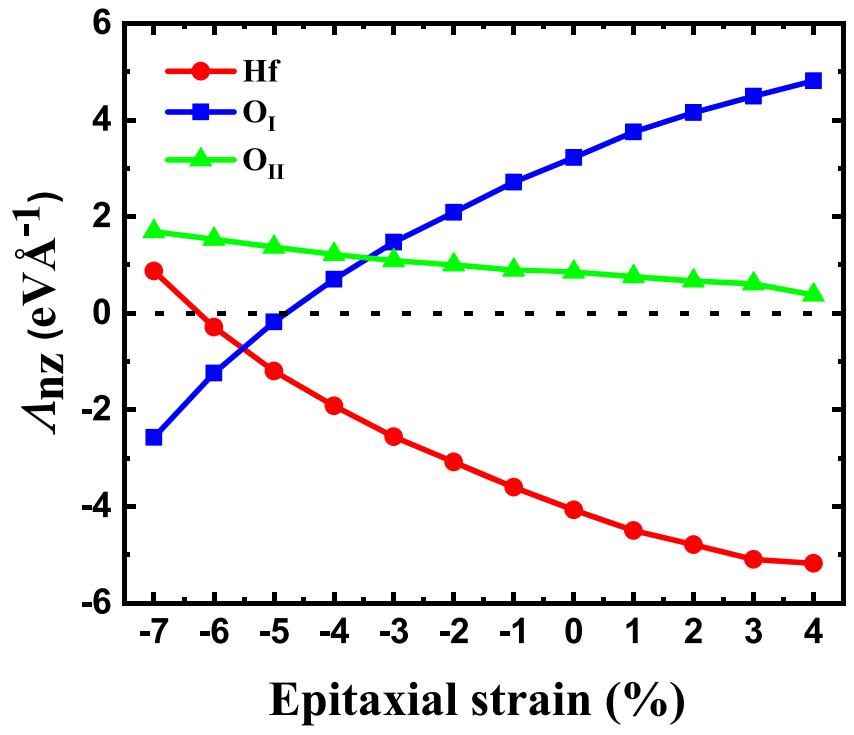

Fig. 7 Epitaxial strain dependence of the $\Lambda$ components that control the $\mathbf{e}_{33}$ response (see text). More precisely, the shown components quantify the third (vertical) component of the force that acts on the $\mathrm{Hf}, \mathrm{O}_{\mathrm{I}}$ and $\mathrm{O}_{\|}$ atoms as a consequence of an applied strain $\eta_{3}>0$.

provided one is able to act upon said atomic environment. Epitaxial strain gives us a control knob to do this.

Let us conclude this part by noting that, as it is obvious from Fig. 6, our calculations predict that it is possible to find epitaxial conditions such that $e_{33}=0$ despite the fact that $\mathrm{HfO}_{2}$ remains polar with space group $P c a 2_{1}$. This result may seem surprising, as the $P c a 2_{1}$ symmetry allows for a non-zero $e_{33}$. However, let us recall that, by itself, symmetry does not tell us anything about the sign or magnitude of $e_{33}$; indeed, a continuous variation of the interatomic interactions responsible for the $e_{33}$ response can change its sign, and even make it vanish, as found here.

Final remarks. Our first-principles analysis thus reveals the atomistic reasons why the predicted longitudinal piezoelectric response of $\mathrm{HfO}_{2}\left(e_{33}\right.$ or $\left.d_{33}\right)$ is negative. More specifically, we show that, when hafnia is strained along its polar axis, the material reacts by shifting the oxygen anions responsible for its spontaneous polarization, so as to best preserve the equilibrium distance of the corresponding Hf-O bonds. Naturally, this atomic rearrangement affects the polarization, in such a way that it grows when the strain is compressive, yielding a negative longitudinal effect.

Guided by this observation, we are able to identify a strategy to tune the piezoresponse -by controlling the chemical environment of the active oxygens-, showing that it can be enhanced or reduced, and even reversed to obtain a positive effect. Admittedly, the specific strategy tested here may not be applicable in practice. (We predict that large compressive epitaxial strains, beyond $-5 \%$, are needed to change the sign of $e_{33}$.) Nevertheless, our qualitative result is important: to the best of our knowledge, this is the first example of a ferroelectric whose piezoelectric response can be reversed by a continuous modification of the lattice, without switching its polarization. This possibility is unheard of among ferroelectrics, and certainly inconceivable in perovskite oxides.

The theoretical prediction of a negative longitudinal piezoresponse is a robust one, corroborated in several ways by us and also obtained by other authors ${ }^{13,14}$. Further, we are not aware of any instance where the theoretical sign of the piezoresponse (as predicted by first-principles methods based on DFT, like the ones 
used here) contradicts the experimental observation. Hence, our experimental ratification of the negative effect -by means of a careful PFM investigation of two reference ferroelectrics (PZT and PVDF) as well as $\mathrm{HfO}_{2}$, all treated in exactly the same way so that a direct comparison can be made- comes as no surprise. The reasonable quantitative agreement between the computed effect (about $-2.5 \mathrm{pm} \mathrm{V}^{-1}$ ) and the one estimated from experiments (between $-2 \mathrm{pm} \mathrm{V}^{-1}$ and $-5 \mathrm{pm} \mathrm{V}^{-1}$ ) further strengthens our confidence in the results presented here. Note that the results of ref. ${ }^{35}$ on pristine $\mathrm{Hf}_{0.5} \mathrm{Zr}_{0.5} \mathrm{O}_{2}$ samples also suggest a negative effect.

Having said this, it is important to recall that the vast majority of published experiments suggest a positive longitudinal piezoresponse $d_{33, \text { eff }} 11,32,33$. Indeed, in the course of this work, we found ourselves that the application of the same experimental protocol to other $\mathrm{HfO}_{2}$ films (thicker, grown by different means) yields a positive longitudinal effect. Hence, we find that different $\mathrm{HfO}_{2}$ samples may present $d_{33 \text {,eff }}$ of different sign. This is a surprising observation, but one that resonates with our prediction that hafnia's piezoresponse can be reversed without switching its polarization. May the differences in the measured sign of $d_{33 \text {,eff }}$ be related to that?

It is thus clear that the experimental question of piezoelectricity in $\mathrm{HfO}_{2}$-based compounds is still open and full of promise. Additional studies will be needed to evaluate how various factors (processing conditions, chemical composition, thickness, mechanical boundary conditions, electrical cycling) affect the outcome. We must try to correlate specific results for $d_{33 \text {,eff }}$ with specific (structural) features in the corresponding samples, distinguishing between intrinsic and extrinsic contributions to the response, a task for which firstprinciples theory may prove a valuable aid to experiment. This is a most appealing challenge, from both fundamental and applied perspectives. On the one hand, it may allow us to understand and master unprecedented ways to control piezoelectricity in ferroelectrics. On the other hand, it may allow us to optimize piezoelectricity in $\mathrm{HfO}_{2}$ up to the point required for applications. We hope the present work will bring impetus to this effort.

\section{Methods}

First-principles simulations. Our calculations are carried out using first-principles DFT as implemented in the Vienna Ab-initio Simulation Package (VASP) ${ }^{39,40}$. We employ the Perdew-Burke-Ernzerhof formulation for solids (PBEsol) ${ }^{41}$ of the generalized gradient approximation for the exchange-correlation functional. In our calculations, the atomic cores are treated within the projector-augmented wave approach $^{42}$, considering the following states explicitly: $5 d, 6 s, 6 p$ for $\mathrm{Pb} ; 3 p, 4 s, 3 d$ for Ti; $2 s, 2 p$ for $\mathrm{O}$; and $5 s, 5 p, 6 s, 5 d$ for Hf. To calculate the response functions we use DFPT ${ }^{22}$. All the calculations (for both $\mathrm{PbTiO}_{3}$ and $\mathrm{HfO}_{2}$ ) are carried out using a plane-wave energy cutoff of $600 \mathrm{eV}$. A $6 \times 6 \times 6 \mathrm{k}$-point sampling of the Brillouin zone ${ }^{43}$ is used for $\mathrm{PbTiO}_{3}$ (corresponding to a 5-atom unit cell), while for $\mathrm{HfO}_{2}$ we use a $4 \times 4 \times 4$ grid (corresponding to a 12 -atom unit cell). The structures are fully relaxed until the residual forces fall below $0.01 \mathrm{eV}^{-1}$ and residual stresses fall below $0.1 \mathrm{GPa}$. We checked that these calculation conditions yield well-converged results.

To verify our predictions for the piezoelectric properties of $\mathrm{HfO}_{2}$, we also run analogous DFPT calculations using the ABINIT first-principles package ${ }^{44}$. In this case, we also consider the Perdew-Burke-Ernzerhof formulation for solids of the generalized gradient approximation for the exchange-correlation functional. We use scalar relativistic norm-conserving Vanderbilt pseudopotentials as implemented in the ABINIT package ${ }^{45}$. In the calculations we treat explicitly the semicore states of $\mathrm{Hf}(5 s, 5 p, 4 f, 5 d$ and $6 s)$ and $\mathrm{O}(2 s)$. We consider a plane-wave cutoff energy of 60 hartree and a $4 \times 4 \times 4 \mathbf{k}$-point sampling of the Brillouin zone. We relax the structures until the residual forces fall below $10^{-6}$ hartree bohr ${ }^{-1}$.

Sample preparation. $200 \mathrm{~nm}$-thick (111)-oriented PZT films with a $\mathrm{Zr} / \mathrm{Ti}$ ratio of $40 / 60$ were fabricated by magnetron sputtering on the Pt bottom electrode. Reactive ion etching was carried out to fabricate capacitors with $50 \mathrm{~nm}$ thick $\mathrm{IrO}_{2}$ top electrodes with lateral dimensions of $80 \times 80 \mu \mathrm{m}^{2}$.

The $20 \mathrm{~nm}$-thick La: $\mathrm{HfO}_{2}$ films were grown by atomic layer deposition on TiN bottom electrodes and capped with a TiN top electrode. The TiN/La: $\mathrm{HfO}_{2} / \mathrm{TiN}$ stack was then annealed in a $\mathrm{N}_{2}$ atmosphere at $800{ }^{\circ} \mathrm{C}$ for $20 \mathrm{~s}$. Details of the growth process for the La: $\mathrm{HfO}_{2}$ films can be found in ref. ${ }^{46}$.
The 12 monolayer-thick (21.6 nm) PVDF films were deposited on Pt/Si substrates by Langmuir-Blodgett methods ${ }^{16}$.

Sample characterization. Switching spectroscopy PFM measurements were performed on a commercial atomic-force-microscopy system (MFP-3D, Asylum Research) in the resonance tracking mode using single-crystalline diamond tips (D80, K-Tek, Nanotechnology) and Pt-coated tips (HQ:DPER-XSC11, MikroMasch). Electrical bias was applied to the top electrode using an external probe, with the frequency of the ac modulation signal around $350 \mathrm{kHz}$ and $650 \mathrm{kHz}$ for the D80 and the Pt-coated tips, respectively. For the PVDF thin films, the conducting tip acted as a local top electrode.

In the DART mode, a feedback loop tracks a shift in the resonance frequency by measuring the difference in the PFM amplitudes for the two drive signals-above and below the resonance frequency. The PFM loops shown in this work were all obtained $3 \mathrm{kHz}$ below the resonance frequency.

\section{Data availability}

All relevant data are included in the figures and tables in the manuscript and in the Supplementary Information file. Additional data (if any) are available from the authors upon reasonable request.

\section{Code availability}

The first-principles simulations were done with VASP, which is proprietary software for which the LIST group owns a license, and ABINIT, which is available at https:// www.abinit.org under the GNU General Public License.

Received: 13 July 2021; Accepted: 11 November 2021; Published online: 15 December 2021

\section{References}

1. Bohr, M. T., Chau, R. S., Ghani, T. \& Mistry, K. The high-k solution. IEEE Spectr. 44, 29-35 (2007).

2. Böscke, T. S., Müller, J., Bräuhaus, D., Schröder, U. \& Böttger, U. Ferroelectricity in hafnium oxide thin films. Appl. Phys. Lett. 99, 102903 (2011).

3. Müller, J. et al. Ferroelectricity in Simple Binary $\mathrm{ZrO}_{2}$ and $\mathrm{HfO}_{2}$. Nano Lett. 12, 4318-4323 (2012).

4. Îñiguez, J., Zubko, P., Luk'yanchuk, I. \& Cano, A. Ferroelectric negative capacitance. Nat. Rev. Mater. 4, 243-256 (2019).

5. Lines, M. E. \& Glass, A. M. Principles and Applications of Ferroelectrics and Related Materials. Oxford Classic Texts in the Physical Sciences (Clarendon Press, 1977)

6. Zhao, X. \& Vanderbilt, D. First-principles study of structural, vibrational, and lattice dielectric properties of hafnium oxide. Phys. Rev. B 65, 233106 (2002).

7. Reyes-Lillo, S. E., Garrity, K. F. \& Rabe, K. M. Antiferroelectricity in thin-film $\mathrm{ZrO}_{2}$ from first principles. Phys. Rev. B 90, 140103 (2014).

8. Delodovici, F., Barone, P. \& Picozzi, S. Trilinear-coupling-driven ferroelectricity in $\mathrm{HfO}_{2}$. Phys. Rev. Mater. 5, 064405 (2021).

9. Lee, H.-J. et al. Scale-free ferroelectricity induced by flat phonon bands in $\mathrm{HfO}_{2}$. Science 369, 1343-1347 (2020).

10. Noheda, B. \& Íñiguez, J. A key piece of the ferroelectric hafnia puzzle. Science 369, 1300-1301 (2020).

11. Schenk, T. et al. Toward thick piezoelectric $\mathrm{HfO}_{2}$-based films. Phys. Status Solidi - Rapid Res. Lett. 14, 1900626 (2020).

12. $\mathrm{Xu}, \mathrm{X}$. et al. Kinetically stabilized ferroelectricity in bulk single-crystalline $\mathrm{HfO}_{2}$ :Y. Nat. Mater. 20, 826-832 (2021).

13. Liu, J., Liu, S., Liu, L. H., Hanrahan, B. \& Pantelides, S. T. Origin of pyroelectricity in ferroelectric $\mathrm{HfO}_{2}$. Phys. Rev. Appl. 12, 034032 (2019)

14. Liu, J., Liu, S., Yang, J.-Y. \& Liu, L. Electric auxetic effect in piezoelectrics. Phys. Rev. Lett. 125, 197601 (2020).

15. Roedel, J. et al. Perspective on the development of lead-free piezoceramics. J. Am. Ceram. Soc. 92, 1153-1177 (2009).

16. Sharma, P., Reece, T. J., Ducharme, S. \& Gruverman, A. High-resolution studies of domain switching behavior in nanostructured ferroelectric polymers. Nano Lett. 11, 1970-1975 (2011).

17. Katsouras, I. et al. The negative piezoelectric effect of the ferroelectric polymer poly(vinylidene fluoride). Nat. Mater. 15, 78-84 (2016).

18. Huan, T. D., Sharma, V., Rossetti, G. A. \& Ramprasad, R. Pathways towards ferroelectricity in hafnia. Phys. Rev. B 90, 064111 (2014).

19. Dutta, S., Aramberri, H., Schenk, T. \& Íñiguez, J. Effect of dopant ordering on the stability of ferroelectric hafnia. Phys. Status Solidi - Rapid Res. Lett. 14, 2000047 (2020)

20. Strukov, B. \& Levanyuk, A. Ferroelectric Phenomena in Crystals: Physical Foundations (Springer, 1998). 
21. Nye, J. F. Physical Properties of Crystals: Their Representation by Tensors and Matrices (Oxford Science Publications, Clarendon Press, 1985).

22. Wu, X., Vanderbilt, D. \& Hamann, D. R. Systematic treatment of displacements, strains, and electric fields in density-functional perturbation theory. Phys. Rev. B 72, 035105 (2005)

23. Haun, M. J., Furman, E., Jang, S. J., McKinstry, H. A. \& Cross, L. E. Thermodynamic theory of $\mathrm{PbTiO}_{3}$. J. Appl. Phys. 62, 3331-3338 (1987).

24. Kvasov, A. et al. Piezoelectric enhancement under negative pressure. Nat. Commun. 7, 1-8 (2016).

25. Damjanovic, D., Budimir, M., Davis, M. \& Setter, N. Piezoelectric anisotropy: Enhanced piezoelectric response along nonpolar directions in perovskite crystals. J. Mater. Sci. 41, 65-76 (2006).

26. Heywang, W., Lubitz, K. \& Wersing, W. (eds.) Piezoelectricity: Evolution and Future of a Technology (Springer-Verlag Berlin Heidelberg, 2008).

27. Gruverman, A., Alexe, M. \& Meier, D. Piezoresponse force microscopy and nanoferroic phenomena. Nat. Commun. 10, 1661 (2019).

28. Hong, S. Single frequency vertical piezoresponse force microscopy. J. Appl. Phys. 129, 051101 (2021).

29. Kholkin, A. L., Akdogan, E. K., Safari, A., Chauvy, P. F. \& Setter, N. Characterization of the effective electrostriction coefficients in ferroelectric thin films. J. Appl. Phys. 89, 8066-8073 (2001).

30. Jesse, S., Lee, H. N. \& Kalinin, S. V. Quantitative mapping of switching behavior in piezoresponse force microscopy. Rev. Sci. Instrum. 77, 0-10 (2006).

31. Furukawa, T. \& Seo, N. Electrostriction as the origin of piezoelectricity in ferroelectric polymers. Jpn. J. Appl. Phys. 29, 675-680 (1990).

32. Böscke, T. S., Müller, J., Bräuhaus, D., Schröder, U. \& Böttger, U. Ferroelectricity in hafnium oxide thin films. Appl. Phys. Lett. 99, 102903 (2011).

33. Starschich, S., Griesche, D., Schneller, T., Waser, R. \& Böttger, U. Chemical solution deposition of ferroelectric yttrium-doped hafnium oxide films on platinum electrodes. Appl. Phys. Lett. 104, 1-5 (2014).

34. Schenk, T. et al. On the origin of the large remanent polarization in $\mathrm{La}: \mathrm{HfO}_{2}$. Adv. Electron. Mater. 5, 1900303 (2019).

35. Chouprik, A. et al. Wake-up in a $\mathrm{Hf}_{0.5} \mathrm{Zr}_{0.5} \mathrm{O}_{2}$ film: A cycle-by-cycle emergence of the remnant polarization via the domain depinning and the vanishing of the anomalous polarization switching. ACS Appl. Electron. Mater. 1, 275-287 (2019).

36. Goldschmidt, V. M. Die gesetze der krystallochemie. Die Naturwissenschaften 21, 477-485 (1926).

37. Brown, I. D. Recent developments in the methods and applications of the bond valence model. Chem. Rev. 109, 6858-6919 (2009).

38. Walsh, A., Payne, D. J., Egdell, R. G. \& Watson, G. W. Stereochemistry of post-transition metal oxides: revision of the classical lone pair model. Chem. Soc. Rev. 40, 4455-4463 (2011).

39. Kresse, G. \& Furthmüller, J. Efficient iterative schemes for ab initio total-energy calculations using a plane-wave basis set. Phys. Rev. B 54, 11169-11186 (1996).

40. Kresse, G. \& Joubert, D. From ultrasoft pseudopotentials to the projector augmented-wave method. Phys. Rev. B 59, 1758-1775 (1999).

41. Perdew, J. et al. Restoring the density-gradient expansion for exchange in solids and surfaces. Phys. Rev. Lett. 100, 136406 (2008).

42. Blöchl, P. E. Projector augmented-wave method. Phys. Rev. B 50, 17953-17979 (1994).

43. Monkhorst, H. J. \& Pack, J. D. Special points for Brillouin-zone integrations. Phys. Rev. B 13, 5188-5192 (1976).

44. Gonze, X. et al. The ABINIT project: Impact, environment and recent developments. Computer Phys. Commun. 248, 107042 (2020).
45. Hamann, D. R. Optimized norm-conserving Vanderbilt pseudopotentials. Phys. Rev. B 88, 085117 (2013).

46. Schroeder, U. et al. Lanthanum-doped hafnium oxide: a robust ferroelectric material. Inorg. Chem. 57, 2752-2765 (2018).

\section{Acknowledgements}

This work was funded by the Luxembourg National Research Fund (FNR) through grants PRIDE/15/10935404 "MASSENA" (S.D. and J.I. ) and FNR/C18/MS/12705883 "REFOX" (H.A. and J.I. .). Work at UNL was supported by the National Science Foundation through EPMD (Grant No. ECCS-1917635) Programs. Work at Namlab was financially supported out of the State budget approved by the delegates of the Saxon State Parliament. We thank Alfred Kersch for insightful comments on the manuscript.

\section{Author contributions}

S.D. performed the first-principles study, assisted by H.A. and supervised by J.I. Samples were prepared by S.G., E.D, C.R., and U.S., and the PFM characterization was carried out by P.B., H.L. and A.G. The manuscript was written by J.I., S.D., A.G., and S.G., with contributions from H.A., U.S., H.L., P.B. and E.D. J.I. conceived and coordinated the work.

\section{Competing interests}

The authors declare no competing interests.

\section{Additional information}

Supplementary information The online version contains supplementary material available at https://doi.org/10.1038/s41467-021-27480-5.

Correspondence and requests for materials should be addressed to Sangita Dutta, Alexei Gruverman or Jorge Íñiguez.

Peer review information Nature Communications thanks Jun Hee Lee and the other, anonymous, reviewer(s) for their contribution to the peer review of this work. Peer reviewer reports are available.

Reprints and permission information is available at http://www.nature.com/reprints

Publisher's note Springer Nature remains neutral with regard to jurisdictional claims in published maps and institutional affiliations.

\begin{abstract}
cC (i) Open Access This article is licensed under a Creative Commons Attribution 4.0 International License, which permits use, sharing, adaptation, distribution and reproduction in any medium or format, as long as you give appropriate credit to the original author(s) and the source, provide a link to the Creative Commons license, and indicate if changes were made. The images or other third party material in this article are included in the article's Creative Commons license, unless indicated otherwise in a credit line to the material. If material is not included in the article's Creative Commons license and your intended use is not permitted by statutory regulation or exceeds the permitted use, you will need to obtain permission directly from the copyright holder. To view a copy of this license, visit http://creativecommons.org/ licenses/by/4.0/.
\end{abstract}

(C) The Author(s) 2021 